\title{
AO corrected satellite imaging from Mount Stromlo
}

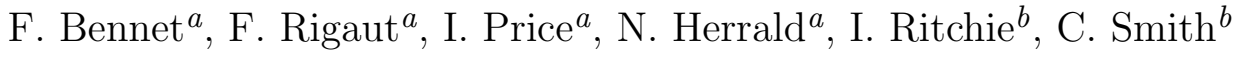 \\ ${ }^{a}$ Research School of Astronomy and Astrophysics, Australian National University, Canberra, \\ Australia; \\ ${ }^{b}$ Electro Optic Systems, Mount Stromlo Observatory, Canberra, Australia
}

\begin{abstract}
The Research School of Astronomy and Astrophysics have been developing adaptive optics systems for space situational awareness. As part of this program we have developed satellite imaging using compact adaptive optics systems for small (1-2 m) telescopes such as those operated by Electro Optic Systems (EOS) from the Mount Stromlo Observatory. We have focused on making compact, simple, and high performance AO systems using modern high stroke high speed deformable mirrors and EMCCD cameras. We are able to track satellites down to magnitude 10 with a Strehl in excess of $20 \%$ in median seeing.
\end{abstract}

Keywords: Adaptive optics, Space situational awareness, Satellite imaging

\section{INTRODUCTION}

Space situational awareness (SSA) is a rapidly growing field for optical telescopes. ${ }^{1}$ The goal of SSA is to track as many orbiting objects as possible, both active satellites and debris in order to avoid collisions and a Kessler syndrome. ${ }^{2}$ These tracked objects are accumulated into a precise catalogue which contains up to date and highly accurate data needed to predict any possible conjuncture. Active satellites can be warned to perform debris avoidance manoeuvres, and possible collisions between debris objects determined in advance.

Orbiting satellites and debris are tracked with a network of radar and satellite laser ranging (SLR) systems around the world. ${ }^{3,4}$ The orbital information for all of these objects is then propagated forward in time to predict any possible close approaches between two objects. ${ }^{5}$ The accuracy of these predictions depends greatly on the precision of the measured orbit, the fidelity of the orbital propagation models, and the shape and mass of the orbiting bodies. SLR systems can provide accurate range data to millimetre precision ${ }^{4}$ for cooperative satellites equipped with retroreflectors. Information on the size and shape of the object is also very important for orbit propagation because orbit perturbations from atmospheric and solar radiation pressure are heavily influenced by object size and shape, particularly if they change with time. ${ }^{6}$ This information when combined with the precise orbit determined with SLR the orbit prediction can reach unprecedented accuracy, assisting in maintaining space situational awareness and avoiding collisions through passive and active measures. ${ }^{7}$

We are developing $\mathrm{AO}$ systems for $\mathrm{SSA},{ }^{8,9}$ and in this paper we present the development and first results of a satellite imaging $\mathrm{AO}$ system for a $1 \mathrm{~m}$ telescope. We focused on making a compact and high performance system using modern high stroke deformable mirror (DM) and low noise high rate EMCCD cameras for imaging and wavefront sensing. We are able to track satellites down to magnitude 10 with an expected Strehl in excess of $20 \%$ in median seeing.

We use the satellite as a natural guide star (NGS) on a Shack-Hartmann wavefront sensor with a closed loop rate of $2 \mathrm{kHz}$. Our system meets the performance requirement of $20 \%$ Strehl at a nominal orbiting altitude of $1000 \mathrm{~km}$, but will also operate on objects down to $600 \mathrm{~km}$. Below this altitude the telescope slew rate is so great that even at $2 \mathrm{kHz}$ the $\mathrm{AO}$ system does not have the bandwidth to provide enough correction. We have developed a system for processing imaging data in real time to remove the need for derotating optics and large stroke tip-tilt correction.

Further author information: (Send correspondence to F. Bennet)

F. Bennet.: E-mail: francis.bennet@anu.edu.au

Adaptive Optics Systems V, edited by Enrico Marchetti, Laird M. Close, Jean-Pierre Véran, Proc. of SPIE Vol. 9909,990911 (C) 2016 SPIE $\cdot$ CCC code: 0277-786X/16/\$18 · doi: 10.1117/12.2232189 

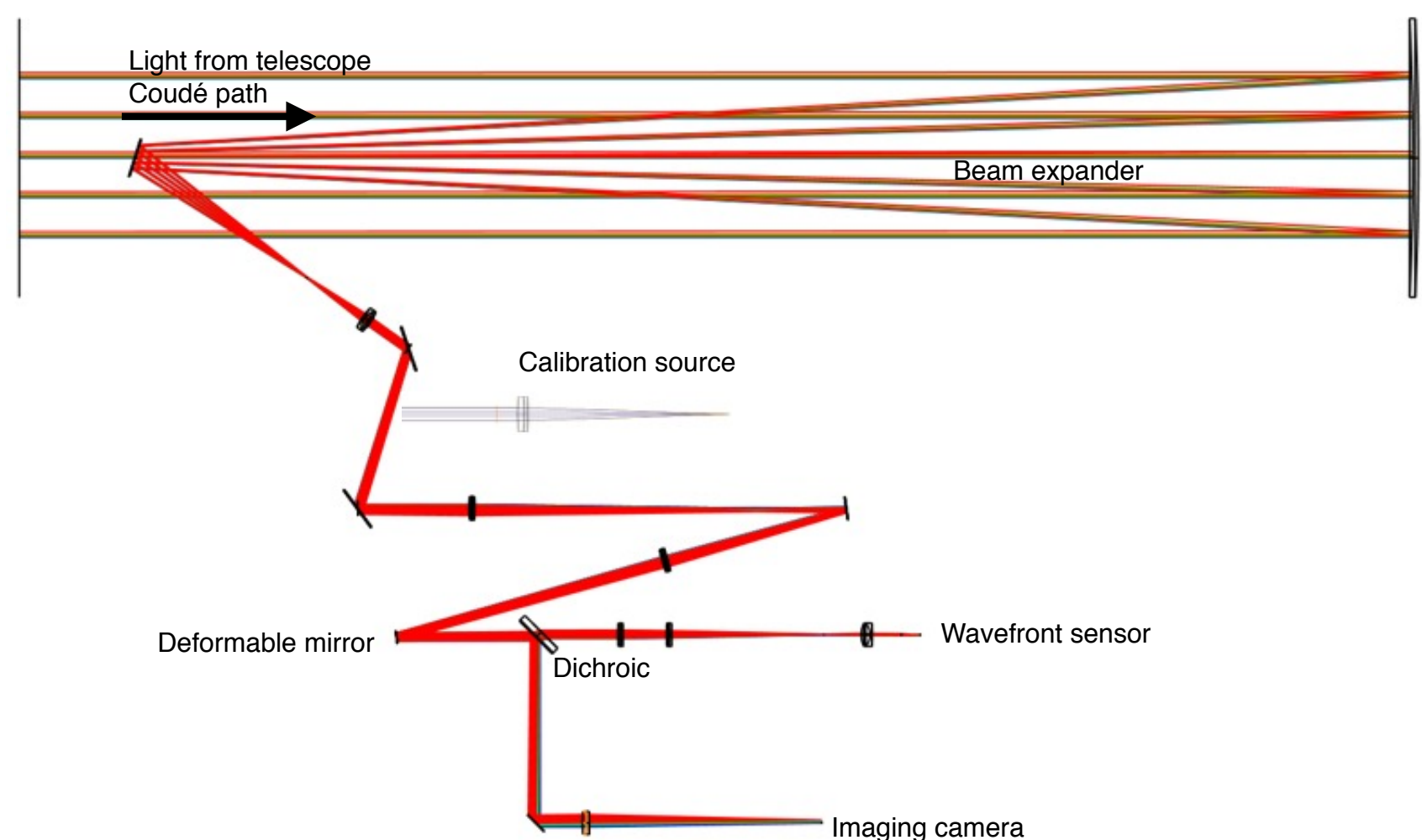

Figure 1. AO System layout. Light from the telescope Coudé path is magnified by a reflective beam expander, and conditioned by optics before reflecting from the deformable mirror. A dichroic beamsplitter splits wavelengths between the wavefront sensor and imaging camera.

We have demonstrated this AO system on a $1 \mathrm{~m}$ SLR telescope at Mount Stromlo Observatory in Canberra, Australia. We present the demonstration results here and plans to further develop the system for a $1.8 \mathrm{~m}$ telescope also located in Mount Stromlo. This next AO system will also be capable of providing orbital information out to geosynchronous orbit using high precision astrometry.

\section{AO SYSTEM DESIGN}

This AO system is designed to image satellites in low Earth orbit (LEO), with a nominal altitude of $1000 \mathrm{~km}$. The requirements for the system are for the system to observe targets with a visible magnitude of 10 up to 1000 $\mathrm{km}$, achieve Strehl ratio of $20 \%$ on a magnitude 10 star in good seeing, with $r_{0}$ of $12-15 \mathrm{~cm}$ and wind speed of $8-10 \mathrm{~ms}^{-1}$.

The layout of the AO system is shown in Fig. 1. Light from the telescope is directed to the AO system as a collimated beam via a Coudé path. A beam expander reduces the beam size from $250 \mathrm{~mm}$ to $12.5 \mathrm{~mm}$, and the pupil is imaged onto the reflective DM face sheet. The light is split with a dichroic beamsplitter, with wavelengths from 450 - $800 \mathrm{~nm}$ being transmitted to the WFS, and 800 - $1000 \mathrm{~nm}$ reflected to the imaging camera. A calibration source is inserted into the system with a mechanical flip mirror, for automated system calibration.

The wavefront sensor is a Shack-Hartmann with $8 \times 8$ subapertures and an OCAM2k camera. The camera can operate at up to $2 \mathrm{kHz}$ with $<0.4 e^{-}$readout noise. We have software selectable lenslet field of view from 7 to 1.2 arcseconds, to accomodate varying seeing conditions. A larger field of view is required to image a larger guide star spot in poor seeing. With a software selectable centroiding window this large field of view does not limit system performance in good seeing, allowing the AO system to operate in a wider range of conditions.

We use an ALPAO DM-69 deformable mirror (DM) with a high speed specification to match the OCAM2k camera frame rate of $2 \mathrm{kHz}$. The large stroke on the DM allows us to correct a large amount of tip-tilt without 

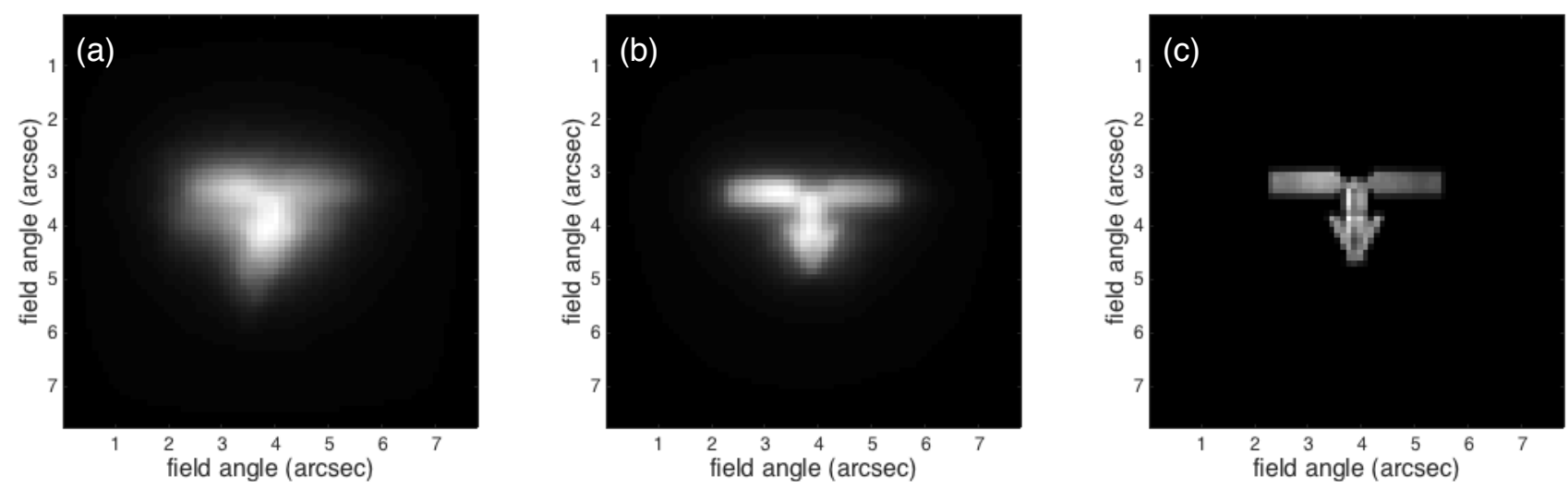

Figure 2. Simulated images of an Iridium satellite taken by $1 \mathrm{~m}$ telescope at $850 \mathrm{~nm}$ under (a) seeing limited with $r_{0}$ of $15 \mathrm{~cm}$, (b) AO correction, and (c) the original image used for the simulation

compromising high-order correction. We are further able to reduce image blue due to tip-tilt by capturing images from the Raptor Falcon EMCCD at a rate of up to $60 \mathrm{~Hz}$, and performing image motion processing on the images before stacking. Capturing images at a high rate has the added benefit of reducing image blur due to satellite rotation. While this rotation means that (typically) only several seconds of images can be stacked, it is possible to fine tune this parameter on a target by target basis for optimum results.

Orbiting objects move at very high rates compared with sidereal objects. With a limited time to acquire and image orbiting bodies our system must make use of all possible data. Capturing images at a high rate allows us to remove field rotation with software, rather than complex and expensive hardware solutions. We stack images from the imaging camera after removing field rotation and image motion, to produce a set of images which can be processed further. We are able to carry out the derotation, image shift and stacking in real time as the images are acquired, and have the option of including a lucky imaging algorithm to only utilise the best frames in a stacked image.

This system is designed for a $1 \mathrm{~m}$ telescope, which has a diffraction limit of 0.18 arcseconds at $850 \mathrm{~nm}$. With AO this telescope will therefor be able to resolve features approximately $85 \mathrm{~cm}$ in size at a range of $1000 \mathrm{~km}$. Satellites such as the Iridium constellation have features on the order of $1-6 \mathrm{~m}$, not including solar panels. Fig. 2 shows simulated images of an Iridium satellite under (a) seeing limit with $r_{0}$ of $15 \mathrm{~cm}$, (b) AO correction, and (c) the original image used for the simulation. The body of the Iridium satellites is approximately $1 \mathrm{~m}$ in width, and $6 \mathrm{~m}$ in length, which subtend an angular size of 0.2 and 1.2 arcseconds respectively at a range of $1000 \mathrm{~km}$.

In order to track satellites with this telescope we must use a lower resolution camera to increase the field of view to acquire the target, with pixels much larger than the Nyquist sampled diffraction limit at $850 \mathrm{~nm}$. This leads us to have much larger resolution elements than we can achieve for stellar objects.

\section{FIRST ON SKY RESULTS}

In Decembee 2016 we operated the system on-sky during the factory acceptance test, using the EOS $1 \mathrm{~m}$ telescope located at the Mount Stromlo Observatory in Canberra, Australia. The goal of the test was to verify system performance against top level requirements. The AO system was first tested on bright stellar sources. Fig. 3 shows images of a magnitude (V band) 3.86 star with (a) the AO loop open, and (b) the AO loop closed. With the AO loop open the FWHM is approximately 2.5 arcseconds due to the poor seeing on the night. With the AO loop closed the light is concentrated in a single core and the first diffraction ring is visible. The closed loop FWHM is 0.27 arcseconds. The images are produced with a very simply lucky imaging algorithm which stacks the brightest $10 \%$ of images, which produces higher Strehl than obtained with an instantaneous image. Image motion is also removed within the lucky imaging algorithm. The signal to noise ratio is much higher in Fig. 3(b) because several images are stacked, and the light is concentrated in only a few pixels. The Strehl ratio of the Fig. $3(\mathrm{~b})$ is $27 \%$ at $850 \mathrm{~nm}$. 


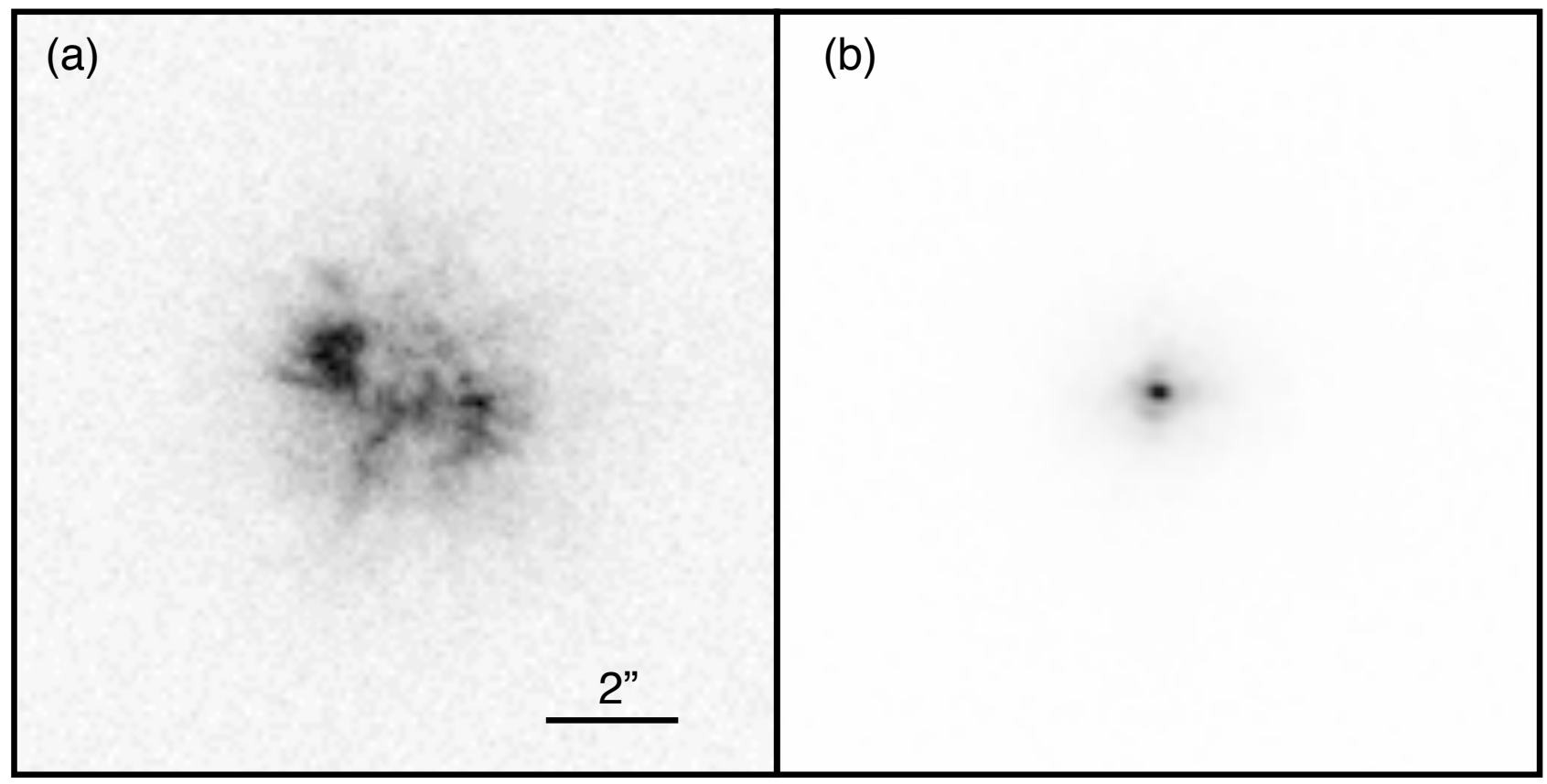

Figure 3. Images of a stellar object with visible magnitude of 3.86. Images of the star with the AO loop (a) open, and (b) closed show the effectiveness of the AO system in compensating atmospheric turbulence.

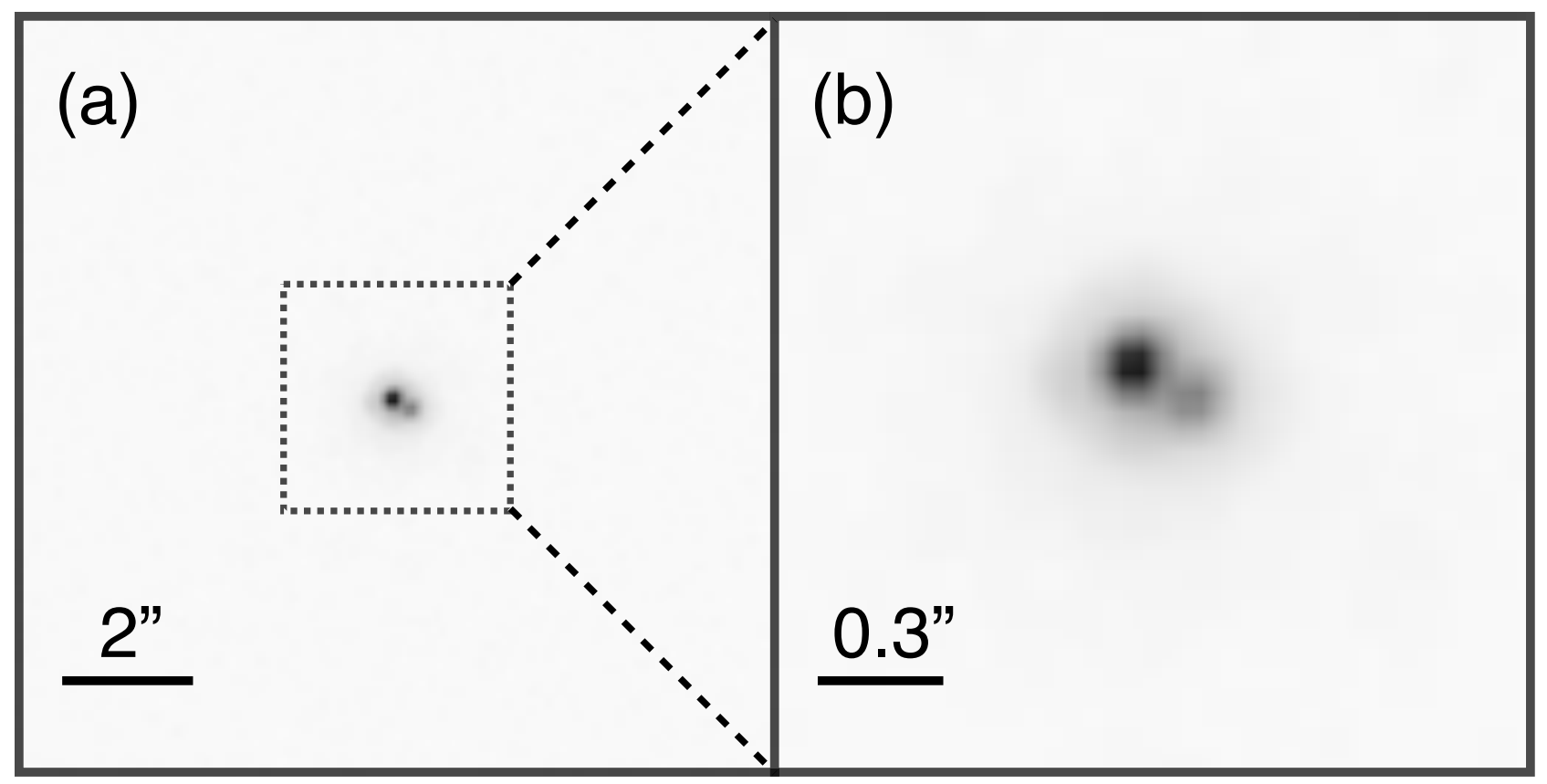

Figure 4. (a) AO Loop closed on a binary star with separation of 0.31 arcseconds, (b) enlarged image clearly shows resolution of both stars. The FWHM of the image is 0.25 arcseconds.

Fig. 4 shows the closed loop resolution of a binary star with a visible magnitude 3.27 . The star separation is approximately 0.3 arcseconds. The image FWHM with the AO loop closed is 0.25 arcseconds.

Fig. 5 shows an image of the Iridium 53 satellite, captured with the AO loop closed. Fig. 5(a) shows the image of the satellite, and Fig. 5(b) shows a cross section through the image. The imaging detector field of view is approximately 23 arcseconds, making acquisition of satellites challenging because their orbits are not known 


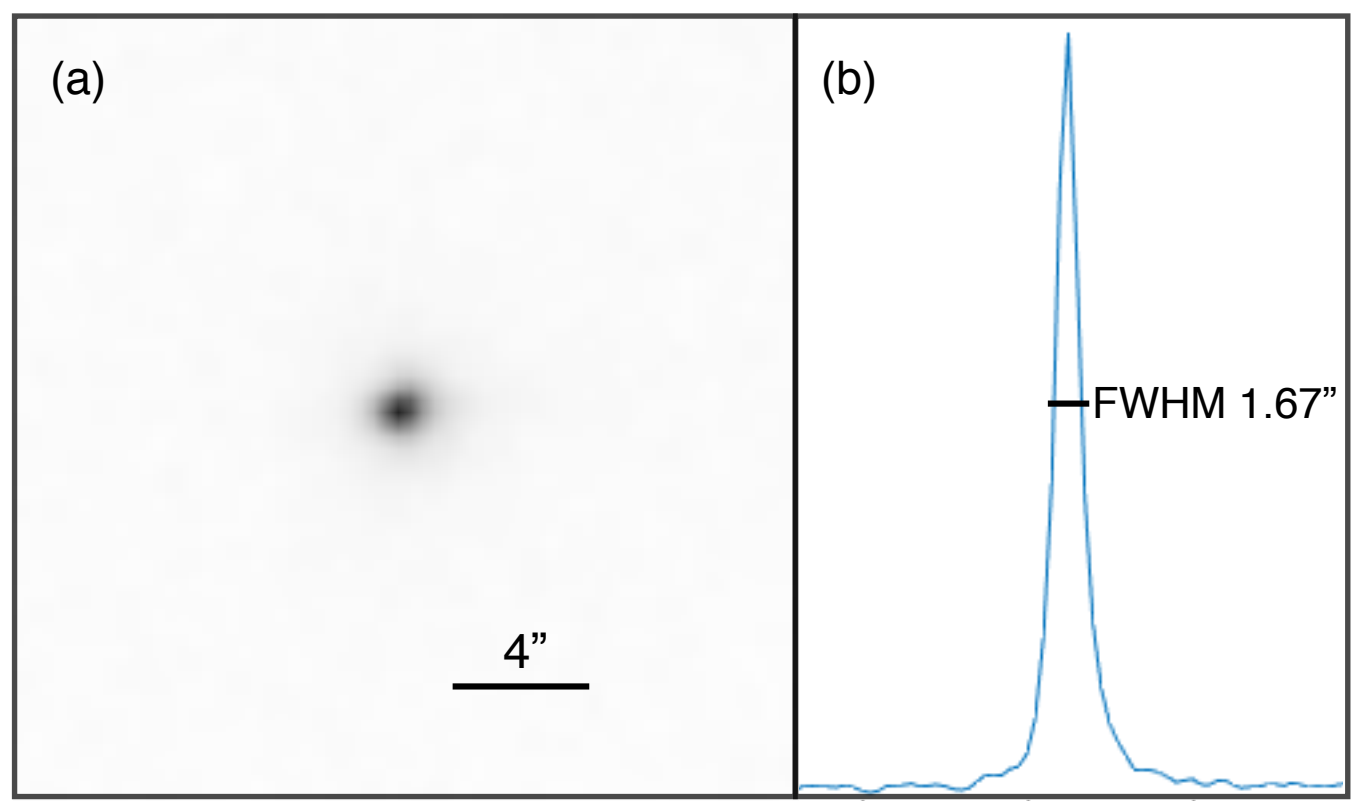

Figure 5. (a) Closed loop image of an Iridium satellite with altitude of approximately $778 \mathrm{~km}$. (b) Image profile with FWHM of 1.67 arc seconds.

precisely enough for the image to fall on the detector. We increased the field of view by increasing the pixel scale of the camera to acquire the satellite and collect data, however this means that satellite features are no longer resolvable. The FWHM was measured to be 1.67 arcseconds, which is considerably larger than the diffraction limit of the telescope, and larger than the FWHM for similar magnitude stellar objects. While the image is symmetric, the measured FWHM is so much larger than other resolved objects we can say with confidence that we have resolved some of the extended source. The larger field of view required to acquire the target limits the resolution of any individual elements of the satellite, however the system does demonstrate that our equipment and technique can resolve satellite features. Future systems plan to use wide field acquisition cameras to allow us to capture images of the satellite at the correct pixel scale.

We were able to demonstrate the systems capability to image faint objects by closing the AO loop on a visible magnitude 9.3 star. In seeing conditions of 2.7 arcseconds we achieved a Strehl ratio of $14 \%$. When compared with simulations under similar conditions we can predict that we will achieve more than the $20 \%$ Strehl requirement under better seeing conditions with $r_{0} 12-15 \mathrm{~cm}$.

\section{FUTURE SYSTEM DEVELOPMENT}

We are currently designing a system for the $1.8 \mathrm{~m}$ telescope operated by EOS at Mount Stromlo in Canberra, Australia. We are aiming for higher performance with this system and to diversify the applications using the larger aperture. The telescope we will be using has wide field acquisition sensors, meaning we will be able to take full advantage of the resolution while tracking satellites. The aim of this system is to characterise the size and shape of objects in LEO, carrying out surveys of LEO objects to assist in building a rich catalogue of orbiting bodies for SSA.

We plan on developing this next AO system to operate in both natural guide star (NGS), and laser guide star (LGS) modes. Using a LGS will enable tracking of fainter objects as the reflected sunlight does not have to be used for wavefront sensing. This system in LGS mode will also be able to track satellites and debris in geosynchronous orbit (GEO) with $1 \mathrm{~m}$ positioning accuracy. Objects at GEO cannot be resolved by a $2 \mathrm{~m}$ aperture, however accurate positioning information can be used to determine accurate orbital elements for SSA.

We are expanding our lucky imaging capabilities to include infra-red imaging technologies such as the SAPHIRA APD detector, which is able to operate at high speed with low readout noise. ${ }^{10}$ The addition of 
lucky imaging and AO for SSA will enhance the performance of the system by producing higher signal to noise images, which are captured at a rate high enough to not be blurred by slow satellite tumbling.

\section{CONCLUSION}

We developed an AO system for imaging satellites in LEO for a $1 \mathrm{~m}$ telescope. The AO system combines wavefront correction at $2 \mathrm{kHz}$ with lucky imaging at up to $60 \mathrm{~Hz}$ to produce high Strehl images at $850 \mathrm{~nm}$. We have demonstrated its capabilities by achieving a Strehl ratio of $27 \%$ in poor seeing of 2.5 arcseconds. We were able to clearly resolve a binary star with a separation of 0.3 arcseconds, with an image FWHM of 0.25 arcseconds. We have demonstrated that we can track resolve satellites while using them as natural guide stars, and demonstrated the AO system performance at close to magnitude 9.3. We continue to develop AO systems for SSA applications, in particular for satellite imaging. We are expanding our AO system capabilities to include laser guide star mode and satellite position to geosynchronous orbit, including more advanced lucky imaging algorithms.

\section{REFERENCES}

[1] Bobrinsky, N. and Del Monte, L., "The space situational awareness program of the European Space Agency," Cosmic Research 48(5), 392-398 (2010).

[2] Kessler, D. J. and Cour-Palais, B. G., "Collision Frequency of Artificial Satellites' The Creation of a Debris Belt," Journal of Geophysical Research 83, 2637 (1978).

[3] Gao, Y., Smith, C., and Greene, B., "Laser Tracking of Space Debris," in [European Space Surveillance Conference], (2011).

[4] Degnan, J. J., "Satellite laser ranging - Current status and future prospects," (4), 398-413 (1985).

[5] Bennett, J. C., Smith, C., Greene, B., Kucharski, D., Rigaut, F., Bennet, F., and Sang, J., "Orbital element generation for an optical and laser tracking object cata- logue," in [16th Annual Advanced Maui Optical and Space Surveillance Technologies (AMOS)], (2015).

[6] Früh, C., Kelecy, T. M., and Jah, M. K., "Coupled orbit-attitude dynamics of high area-to-mass ratio (HAMR) objects: Influence of solar radiation pressure, Earth's shadow and the visibility in light curves," Celestial Mechanics and Dynamical Astronomy 117(4), 385-404 (2013).

[7] Mason, J., Stupl, J., Marshall, W., and Levit, C., "Orbital debrisdebris collision avoidance," Advances in Space Research 48, 1643-1655 (nov 2011).

[8] Bennet, F., Conan, R., D’Ogreville, C., Dawson, M., Paulin, N., Price, I., Rigaut, F., Ritchie, I., Smith, C., and Uhlendorf, K., "Adaptive Optics For Laser Space Debris Removal," in [Adaptive Optics Systems III], 844744(1-6) (2012).

[9] Bennet, F., D’Orgeville, C., Gao, Y., Gardhouse, W., Paulin, N., Price, I., Rigaut, F., Ritchie, I., Smith, C., Uhlendorf, K., and Wang, Y., "Adaptive optics for space debris tracking," in [SPIE 9148, Adaptive Optics Systems IV], (2014).

[10] Atkinson, D., Hall, D., Baranec, C., Baker, I., Jacobson, S., and Riddle, R., "Observatory deployment and characterization of SAPHIRA HgCdTe APD arrays," Proceedings of SPIE 9154(808), 915411 (2014). 